\title{
Syllabus for postgraduate specialisation in Nuclear Medicine
}

\author{
2006/07 update
}

\author{
Alberto Cuocolo • Metka Milčinski • \\ Angelika Bischof Delaloye
}

Published online: 22 December 2009

(C) EANM 2010

\section{Nuclear medicine training in the European Union}

Nuclear Medicine (NM) became an independent medical specialty in the European Directives in 1988. The minimum duration of the postgraduate specialized training in the European Union is 4 years, but may be extended beyond this period according to the requirements of training in other clinical disciplines.

Candidates for specialized training should have a good general background in internal medicine. More detailed knowledge has to be acquired of those conditions which may need to be investigated or treated by NM techniques, as well as of some complementary methods as far as they relate to NM procedures. Training in basic sciences is

The authors wrote this article on behalf of the European Board of Nuclear Medicine.

\footnotetext{
A. Bischof Delaloye $(\bowtie)$

Nuclear Medicine Department,

University Hospital, Bugnon 46,

1011 Lausanne, Switzerland

e-mail: angelika.bischofdelaloye@chuv.ch

M. Milčinski

Department of Nuclear Medicine,

University Medical Center, Zaloška 7,

1525 Ljubljana, Slovenia

e-mail: metka.melcinski@kclj.si

A. Cuocolo

University of Naples - Federico II,

Department of Biomorphological and Functional Sciences,

Via Sergio Pansini 5,

IT-80131 NAPOLI, Italy

e-mail: cuocolo@unina.it
}

required, such as pharmacokinetics, radiochemistry, instrumentation, computer science and quality control.

The quality of training has to be objectively assessed, using final examination on a national basis covering basic sciences and clinical skills, after satisfactory completion of a minimum number of courses and/or workshops and a formally organized and controlled practical training. Each training programme should contain a standard against which the progress of the trainee can be assessed for each element of the syllabus. The assessment may take the form of an interview, a written paper, an essay, a set of multiple-choice questions, or an oral examination of displayed images of various NM techniques in clinical practice. Continuous assessment is another alternative. Each end of year assessment should carry a score that indicates how the candidate has progressed against the set target. Successful trainees are awarded with a final certificate, degree or diploma that is recognized by the government, local health authority and hospital as an assurance of specialist competence in NM.

The clinical training of physicians specializing in NM should include: 1) a minimal theoretical foundation of the general principles of $\mathrm{NM}$ with active participation in clinical presentations, seminars and meetings and 2) in vivo diagnostic procedures. Personal responsibility (including indication, performance and interpretation) must be taken for at least 3.000 in vivo NM diagnostic procedures, with a broad-spectrum of the most currently used procedures. The list of the procedures published in the syllabus will be subject to revision. It is recommended that a period of training be spent away from the main department in at least one other recognized centre. In addition the training should cover therapy with unsealed radioactive sources. The trainee must have taken part in a sufficient number of 
Table 1 Responsibilities of the nuclear medicine specialist

Define the patient's and clinician's rationale for the request or referral Determine and organize the appropriate tests and protocols

Adapt the protocols to the needs and condition of the patient

Assess and accomplish interventions (physiological, pharmacological or mental stress related)

Regulate the study analysis and interpretation according

to the clinical information

Interpret the results and their clinical, biological and pathological

implications

Consider follow-up consultations

Guarantee the safety of both the patient and staff

Provide training and education for junior doctors and technical staff

various NM therapeutic procedures. The responsibilities of the NM specialists are listed in Table 1.

I. Scope and limits of the medical specialty of Nuclear Medicine

1. Definition (scope):

NM utilizes the nuclear properties of matter to investigate disorders of metabolism and function, of physiology and pathophysiology, and of anatomy to diagnose and treat disease with unsealed sources of radioactivity. The range of procedures that are implicit within this definition include in vitro procedures, in vivo imaging with radiopharmaceuticals and other techniques related to nuclear physics in medicine as well as the medical applications of radiobiology, dosimetry and radiation protection.

2. Clinical knowledge and experience:

A good general background in medicine (internal medicine, surgery) is assumed. More detailed knowledge is required of those conditions which may need to be investigated or treated by NM techniques.

3. NM may also make use of complementary methods related to NM procedures. These include:

- Ultrasound

- ECG (including dynamic + pharmacological stress testing) and management of emergencies in cardiac NM

- Fine-needle biopsy

- Quantitative imaging: MRI, MRS, and correlative imaging methods (including CT)

- Spirometry

- Non-radioactive laboratory assays

- Bone densitometry

- Other available techniques complementary to NM procedures

- CT for attenuation correction and anatomic localization
4. NM specialists may cooperate in the assessment, prevention and treatment of physical or medical accidental contamination or incorporation of radionuclides.

II. Formative objectives

1. Basic theoretical formative objectives:

- Mathematics and physics, including radiation physics and statistics

- Informatics

- Kinetic modeling

- Imaging processing

- Instrumentation

- Interaction between radiation and biological structures

- Radiobiology

- Radiation protection and radiopathology

- Physiology and pathophysiology

- Clinical radiopharmacology

2. Specific theoretical formative objectives:

- Evaluation and statistics of radioactive counting

- Radiochemistry, radiopharmacy and quality control of radiopharmaceuticals

- Radionuclide labeling of cells, sub cellular structures and biological molecules

- Applications of radiotracers: indications, procedures and results, methodology and dosimetry

- Data acquisition and processing techniques for imaging treatment, including SPECT and PET

- Integration and evaluation of the diagnostic results with the clinical data and the results of other procedures

- Radiation protection

3. Integrative objectives:

- Radiopharmaceutical preparation

- Radiopharmaceutical administration to the patients

- Diagnostic imaging methodology in vivo for different organs and systems

- Study of neoplastic and non-neoplastic pathological processes with radionuclides and radiopharmaceuticals in vivo

- Application of radionuclide therapy to neoplastic and non-neoplastic pathological processes

- Principles and applications of radioimmunological and immunoradiometric techniques in vitro

- Principles of other diagnostic imaging techniques (including ultrasonography, CT, MRI, digital radiology)

- Integration and interpretation of the results of the NM procedures with those of radiodiagnostic methods, with particular attention to fusion imaging modalities 
- Basic principles of scientific research methodology

- Organization and management of a NM service and basic methodology for programming scientific research

- Study of the legislation related to the transportation, storage, disposal and use of radioactive material

III. Basic sciences training (Syllabus)

This section provides an indication of training for each of the four years:

Year 1 (a) Scientific principles:

- Basic physics and mathematics;

- Instrumentation;

- Principles of computer science;

- Basic radiation biology and radiation protection;

- Basic radiopharmacy and radiochemistry;

- Principles of tracer technology.

(b) Clinical NM:

- Diagnostic imaging: mode of pharmaceutical uptake; normal and abnormal appearances of images, normal variants and common artifacts in bone, heart, lung, kidney, brain, thyroid, tumor and infection images.

- Therapeutic applications: basic principles of radionuclide therapy; treatment of hyperthyroidism, thyroid cancer and metastatic bone pain.

- Principles of radiation protection: ALARA (as low as reasonably achievable), ALARP (as low as reasonably practicable).

- Diagnosis and treatment of thyroid diseases.

Year 2 (a) Requirements of Year 1 in greater depth:

- Tracer kinetics;

- Computing and image processing;

- Radiobiology including the biological effects of high and low levels of radiation;

- Linear hypothesis and the threshold hypothesis of the biological response to low level radiation;

- The effective dose equivalent and the calculation of radiation dose from radiopharmaceuticals.

(b) Radiopharmacy:

- Properties of commonly used diagnostic and therapeutic radiopharmaceuticals;

- Production of radionuclides by reactors, cyclotrons and radionuclide generators;

- Quality assurance and quality control of radiopharmaceuticals.

Year 3 (a) Requirements of Year 2 in greater depth:

- Principles of radiology including dual energy X ray absorption (DEXA), ultrasound, CT and MRI imaging;
- Learning of cross-sectional anatomy;

- Correlative imaging of NM images and those from other imaging techniques;

- Special diagnostic investigations in cardiology, lung disease, gastroenterology, hepatobiliary diseases, nephro-urology, neurology and psychiatry, endocrinology, hematology, oncology and infection.

(b) Therapeutic applications:

- Treatment of bone metastases, neuroendocrine tumours and other malignancies as well as polycythemia;

- Use of radiolabeled monoclonal antibodies and peptides for tumor therapy.

Year 4 Further practice and experience of techniques learned in years $1-3$ :

- Ethics;

- Legal and regulatory requirements;

- Audit;

- Departmental and hospital management;

- Research techniques and evaluation;

- Teaching and training.

The quality of the basic science training has to be objectively assessed, using the following methods:

1. Final examination (covering basic science and clinical knowledge) on national basis and/or

2. Satisfactory completion of accredited, regional or national (international) courses or workshops in different fields (physics etc.): 120 hours recommended.

Radiation protection and regulatory issues have to obey local/national requirements.

\section{Clinical training in NM}

A clinical training has to be added to the courses and has to be formally controlled. The clinical training of physicians specializing in NM should include theoretical and practical training within and outside of the NM Department. Minimum standards are indicated here.

1. Theoretical founding in $N M$

A minimum of 30 hours of formal description of general principles of NM procedures is required. Active participation in clinical presentations, seminars and meetings is recommended.

2. Practical training in NM

Postgraduate trainees are obliged to play an active inservice role in the practice of NM in order to familiarize themselves with all the techniques required of a NM practitioner, such as: 
- Protocols of in vivo and therapeutic procedures;

- Data acquisition and processing with various types of equipment, quality control of instruments and labeled agents;

- Interventional procedures, including physiological, pharmacological and mental stress related for diagnostic applications, and all therapeutic interventions;

- In vitro protocols and procedures, as appropriate.

3. In vivo diagnostic procedures

At the end of the training program, postgraduate trainees must be capable to plan, perform, report and archive diagnostic procedures in vivo related to the following clinical areas:

- Central nervous system

- Bone and joints

- Cardiovascular system

- Respiratory system

- Gastrointestinal system

- Urinary and genital system

- Endocrine system

- Haematopoetic and lymphatic system

- Neoplastic processes

- Inflammatory and degenerative processes

The trainee must reach a total of 3,000 documented diagnostic procedures. The minimum recommended number for each procedure is as follows:

a) Central nervous system $100(80 \%$ SPET or PET)

b) Bone and joints 600

c) Cardiovascular system 500 (80\% SPET or PET)

d) Respiratory system 300 (50\% combined V/Q)

e) Gastrointestinal system 50

f) Urinary and genital system 400

g) Endocrine system 400

h) Haematopoetic and lymphatic system 50

i) Neoplastic processes $550(80 \%$ SPET, PET or SPET/CT, PET/CT)

j) Inflammatory and degenerative processes 50

It is recommended that at least 100 procedures have been performed in pediatric patients. Some flexibility may be accepted, but a broad-spectrum of most currently used procedures has to be covered. This list will be subject to periodic revision. It is recommended that a period of training is spent away from the main department in at least one other recognized training centre.

4. Clinical training in addition to $N M$

Clinical bedside training in a clinical specialty is required before or during specialist training. A limited period of 6 months in diagnostic radiology or clinical physiology may replace a clinical bedside semester.
The proportion of the total training period devoted to clinical training and to NM may vary according to various factors, among them the total length of the training. The minimum advisable duration of training is 5 years. In some countries total duration of the specialized training is 4 years (which corresponds to the minimum duration in the Directive of the EC). In any case a minimum of 3 years should be devoted to NM exclusively and 1 year to other specialties. In countries where part of the requested training is provided during rotation in other departments it must be made sure that all requirements of the syllabus are fulfilled.

5. In vitro procedures

Training can also cover in vitro procedures including analysis with (radio-)immunological methods, quality control and interpretation, with a recommended minimum duration of 3 months.

6. Therapy

Training should include indication, administration, and therapeutic applications of radionuclides, dosimetry, radiation protection and follow-up after therapy.

The trainee must have taken part in 100 various NM therapeutic procedures.

7. Clinical audit

The trainee should have received education in audit of clinical NM and in the administration and financing of a NM Service.

8. Function as expert

The trainee must acquire legal expertise in health care problems related with unsealed radionuclides.

V. Assessment of training in NM

Since trainees will take over the responsibilities of a nuclear physician, they must pass a qualifying test that covers both theoretical knowledge and practical abilities in the daily practice of NM. A board or similar form of authority will award a certificate to successful trainees.

\section{Appendix}

The aim of the syllabus is to set standards, made of duration and content (qualitative and quantitative, practical and theoretical) of training in nuclear medicine. These essential requirements can be adapted to local practice and necessities to fit the specific situation of each country. The content of the syllabus also represents the standard for accreditation of training centres and the basis of the Fellowship exam. 